\title{
Haemodynamic study of Flow in concentric and eccentric stenosed carotid Artery
}

\author{
S. M. Abdul Khader ${ }^{1}$, Raghuvir Pai ${ }^{2 *}$, Ganesh Kamath ${ }^{3}$,Mohammed Zuber ${ }^{4}$ and V. R. K. \\ $\mathrm{Rao}^{5}$ \\ ${ }^{1}$ Department of Mechanical Engineering, School of Science and Engineering, Manipal International \\ University, No.1, Persiaran MIU, Nilai-71800, Malaysia, \\ ${ }^{2}$ Department of Mechanical and Manufacturing Engineering, Manipal Institute of Technology, \\ Manipal Academy of Higher Education, Manipal, 576104, India \\ ${ }^{3}$ Department of Cardiothoracic and Vascular Surgery, Manipal Academy of Higher Education, \\ Manipal, 576104, India \\ ${ }^{4}$ Department of Aeronautical and Automobile Engineering, Manipal Academy of Higher Education, \\ Manipal, 576104, India \\ ${ }^{5}$ Krishna Institute of Medical Sciences, Secunderabad, 500 003, India
}

\begin{abstract}
Study of flow through arteries is gaining importance in the recent times due to onset of several cardio-vascular diseases like atherosclerosis (block in artery), aneurysms (bulging of artery), hypertension etc. The application of CFD will be useful in demonstrating the underlying mechanism of flow past the diseased arteries. In the present study, initially an approximate eccentric and concentric 50\% stenosed carotid model is generated in ANSYS 12.0 based on patient data obtained from ultrasound Doppler scan. Later the percentage of stenosis is increased to $60 \%, 70 \%, 80 \%$ and $90 \%$ in both the cases. A transient analysis has been performed for several pulse cycles in ANSYS FLOTRAN for various percentage cases. The results obtained from ECN and CCN cases are compared to observe the changes in flow behavior in the downstream of stenosis as compared with normal case. The formation of vortices and flow separation zone in downstream in eccentric case is higher than in concentric case. Comparison of results concludes that, with the increase in severity of stenosis, the flow changes abruptly causing an increase in velocity and WSS at throat region. The simulation results obtained agree well with clinically observed data and available literature.
\end{abstract}

Keywords: CFD; Stenotic flow; Common Carotid Artery; ANSYS APDL

\section{Introduction}

The high level of maturity reached in many computational disciplines, prompts for the study of new multidisciplinary applications of great importance for engineering purposes. Such is the case of blood flow analysis through arteries. The flow analysis can help in

\footnotetext{
*Corresponding author: raghuvir.pai@manipal.edu
} 
understanding the flow through elastic arteries. The CFD tools can be more promising in analysing such complex flow.

Besides theoretical work, experimental studies and clinical measurements have advanced the understanding in the past two centuries [1]. While experiments can be expensive or measurement data hard to attain, properly validated coupled computational simulations can hold the answer in characterizing conditions, such as flow and pressure and also to understand arterial flows [2]. This type of study can be extended in the applications such as disease research, where fluid mechanical conditions are correlated to regions prone to atherosclerosis, medical device design where the interactions between a device and the blood stream are modelled [3]. The study of stenosis, which is a constriction in the blood vessels, can be helpful in understanding the biomechanics of vascular diseases. The flow behavior in the stenosed artery is quite different than one in the normal arteries $[4,5]$.

The concepts of fluid dynamics can be applied to study the interesting aspects of generation, detection and the treatment of stenosis [6]. The stenotic geometry is highly complex, but in the preliminary study it can be simplified as a constriction in a cylindrical tube. Generally the flow through the constricted tube is characterized by high velocity jet generated in constricted section and flow separation downstream to the stenosis $[3,7]$. The constriction can be broadly classified as symmetric-concentric and asymmetric-eccentric conditions. In case of concentric condition, a high speed jet of fluid extends downstream along the centerline of model, whereas in case of eccentric condition, the flow pattern is almost stagnant and forces the vorticity against the wall while flowing in downstream causing high flow separation [8].

An attempt has been made in the present study to demonstrate the significant changes for concentric and eccentric conditions with various stenosis percentages starting from $50 \%$ in steps of $10 \%$ till occlusion. This study focuses on detailed investigation of flow parameters like velocity, WSS, pressure contours. The comparison of results obtained reveals the high WSS zones on the far wall of downstream of the throat causing degradation of wall tissue $[3,8]$. Also shear layer dissipates more quickly due to formation of high eddies in downstream [9].

The flow behavior changes abruptly with the increase in velocity at throat and large recirculation in the downstream of stenosis. This type of study will be useful to predict the outcome of severity of stenosis, and aids the physicians with better insight into flow dynamics. Moreover it is also observed that severity less than $75 \%$ is considered as mild and anything greater than this is termed as critical $[4,10]$. This type of study will be useful to predict the outcome of severity of stenosis, and aids the physicians with better insight into flow dynamics.

\section{Methodology}

\subsection{Governing Equations}

In the present study, fluid model is assumed to be incompressible Newtonian fluid to minimize the complexity of analysis and modelled with a density of $1500 \mathrm{~kg} / \mathrm{m}^{3}$ and dynamic viscosity of 0.04 poise[5,6]. The dynamic flow is governed by the continuity and Navier-stokes equations $[11,12]$, respectively as

$$
\nabla v=0
$$




$$
\rho\left(\frac{\partial v}{\partial t}+v \nabla v\right)=-\nabla p+\mu \nabla^{2} v
$$

where $v$ is the velocity vector, $\rho$ is the density and $\mathrm{P}$ is the pressure.

\subsection{Modelling}

The present study is carried out considering the realistic physiological conditions. The basic parameters required for the modelling purpose is done using Doppler scan on a healthy young male volunteer [13]. The B-mode scan is used to obtain velocity spectrum and Mmode scan is obtain geometry details [14]. Based on the date obtained, an approximate eccentric model and concentric model having a constriction ratio of $50 \%$ and length of $18 \mathrm{D}$ is generated in ANSYS 12.0. Fig. 1 shows the eccentric model where the stenosed region is encircled and similarly the concentric model is shown in Fig.2.

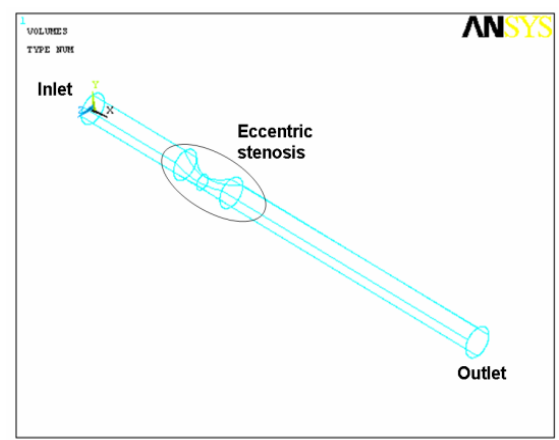

Fig. 1. Eccentric stenosed model

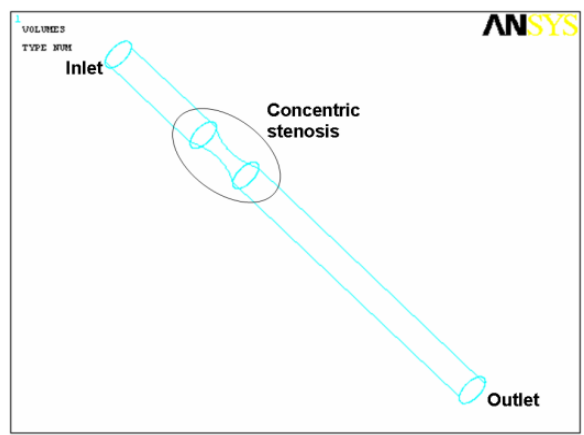

Fig.2. Concentric stenosed model

In order to analyse the effect of severity, the initial $50 \% \mathrm{ECN}$ and $\mathrm{CCN}$ stenosis is increased and separate models are generated for $60 \%, 70 \%, 80 \%$ and $90 \%$ stenosis and the rest of the parameters remain same for all the later cases. This will yield useful simulation data when the stenotic severity progresses, which will be useful in quantifying haemodynamic factors that indicates plaque, rupture potential.

\subsection{Analysis}

An APDL is written in ANSYS to generate the model and mesh with 3D brick element which are discretised into approximately 150000 tetrahedral elements for both the cases [15]. The time varying velocity is applied at inlet as shown in fig.3, constant pressure boundary condition at outlet and no-slip condition is considered along the walls. The density and dynamic viscosity of the blood considered are $1050 \mathrm{~kg} / \mathrm{m} 3$ and $0.004 \mathrm{~N}-\mathrm{sec} / \mathrm{m} 2$ respectively $[14,15]$. 


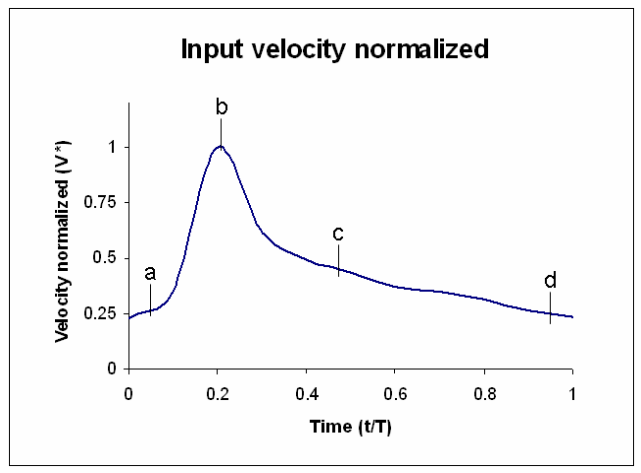

Fig.3. Concentric stenosed model

The analysis has been performed for 3 pulse cycles and each cycle is discritised into 80 steps. In the time varying velocity applied, the following regions of interest are highlighted. Region a - refers to early systole, Region $\mathbf{b}$ - refers to peak systole, Region $\mathbf{c}$ - refers to early diastole, Region $\mathbf{d}$ - refers to late diastole

\section{Results and Discussion}

A CFD study has been carried out to evaluate the effect of stenosis on the flow behavior. The results obtained from the various stenotic percentages for both eccentric and concentric models have been presented. The velocity contours, WSS and pressure have been considered in the present study for comparing the deviation in the flow. In the high grade stenosis, the flow resistance in arteries increases, which forces the body to raise the blood pressure to maintain the necessary blood supply [4]. Both the high pressure and the narrowing of blood vessel cause high flow velocity, high shear stress and low or negative pressure at the throat of the stenosis, low shear stress and flow separation at the distal side of the stenosis [5]. 


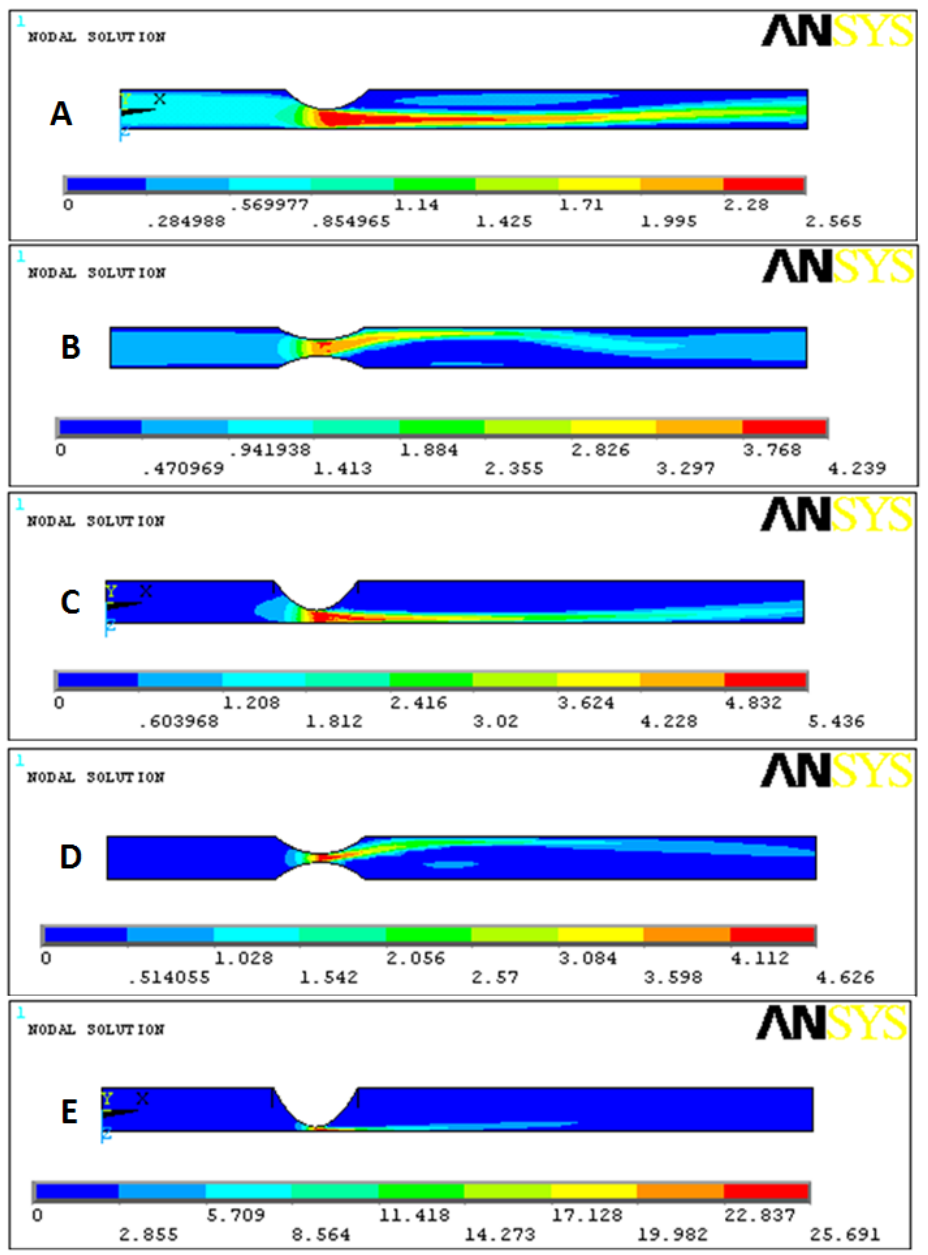

Fig.4. (a) $50 \mathrm{ECN}$ peak systole (b) $60 \mathrm{CCN}$ early diastole (c) $70 \mathrm{ECN}$ early systole (d) $80 \mathrm{CCN}$ end diastole (e) $90 \mathrm{ECN}$ peak systole

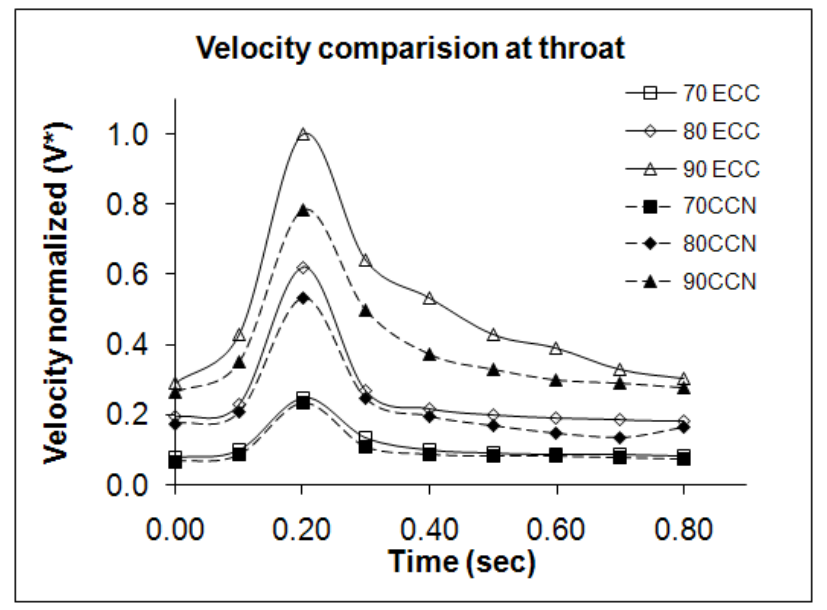

Fig.5. Velocity comparison at the throat 


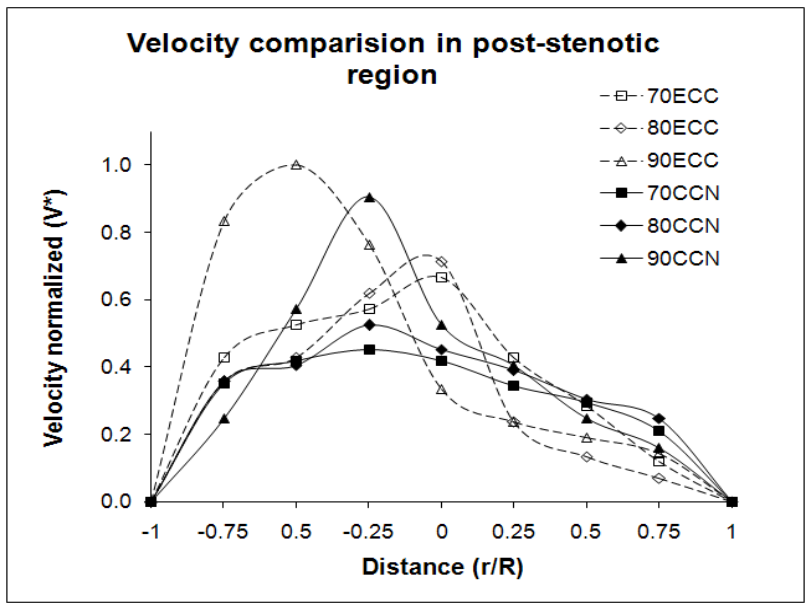

Fig.6. Velocity comparison in post-stenotic region

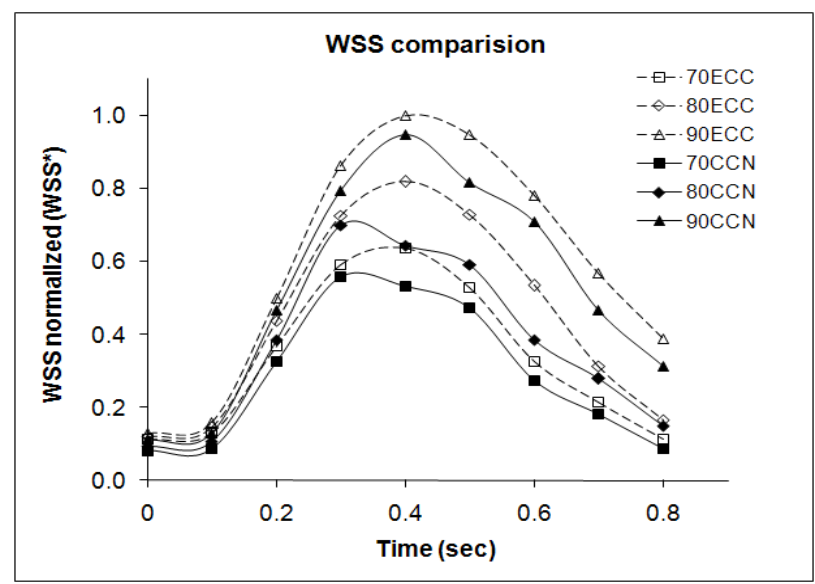

Fig.7. Wall shear stress comparison

Fig 4 shows the velocity profile for different values of stenosis during various instants of pulse cycle. The velocity contours depicts interesting flow features that varies throughout the pulse cycle. The flow behavior is almost similar in all the cases upstream of stenosis, whereas complex changes are observed in the distal side [3, 6]. Fig. 4(A) shows the velocity profile during peak systole for $50 \%$ of stenosis. Due to eccentric type, a flow jet is formed at the throat which extends along $3 \mathrm{D}$ times in downstream end. The flow separation is observed in the immediate vicinity in downstream side. Due to concentric type of stenosis as seen from Fig. 4(B) for $60 \%$, the vortex formation is observed in the distal side extending for $2.5 \mathrm{D}$ times during early diastole. The flow separation becomes more complex during early diastole due to flow decelaration.

It can be inferred from the fig. 4(C) and 4(E), that as the percentage of stenosis increases, the constriction of flow increases resulting in lesser flow rate on the downstream side. Similarly for CCN case as seen in Fig. 4(D) during end diastole, the formation of flow jet still persists in downstream end inspite of flow decelaration. Since the stenosis greater than $75 \%$ is considered critical, a comparison of velocity at throat for all the ECN and CCN percentages greater than $75 \%$ are compared as shown in the Fig.5. It is observed that for $90 \%$, the velocity increases instantly at the ECN than at the CCN. The difference in velocity during peak systole between $\mathrm{ECN}$ and $\mathrm{CCN}$ is maximum when compared for less 
critical cases like $80 \%$ and $70 \%$. During the later part of pulse cycle, in less critical cases, the difference in flow is less than those compared with more critical cases. The flow dynamics in stenosis is characterised by instant increase of velocity at throat and flow jet extending to certain distance in downstream side. The flow jet subsides after certain distance causing flow separation and formation of eddies in distal end. The flow behaviour is non-uniform throughout the pulse cycle in post-stenotic region as seen from Fig.6. In case of $90 \%$, the flow jet in ECN still exists extending to more length than CCN. The flow orientation towards wall is more prominent in ECN than in CCN [6]. As percentage of stenosis decreases, the flow jet length subsides and flow orientation in $\mathrm{CCN}$ is more towards core, but in ECN it is still extending towards boundary.

As the severity of stenosis increases, the WSS also increases as observed from the comparison of WSS shown in Fig.7 for more critical cases. It can be observed that, during early pulse cycle, there are no significant changes in WSS pattern. Irrespective of the severity, the WSS is observed to be maximum during post peak phase in all ECN cases which is due to existence of flow jet after peak phase of cycle. In case of CCN, maximum WSS shifts before ECN condition and it immediately decreases because of sudden drop of flow jet in distal side. The result obtained agrees well with the clinical observations and also with available literature. An instantaneous increase in velocity is observed during the peak systole at throat region and with jet flow extending into the post-stenotic region. The flow behavior in case of ECN at the downstream of stenosis is characterized by flow separation leading to formation of eddies and in the case of CCN, the flow jet extends to larger length causing vortices. This behavior gets complex as the severity of stenosis increases [4, 5]. Further, the hypothetical cases of increased severity were used in prediction of changes in flow behavior as the percentage of stenosis increases. This can be beneficial in the prognosis of the clinical cases.

\section{Conclusions}

Present study demonstrates the major flow changes for eccentric and concentric stenotic condition. The flow is maximum during peak systole of the pulse cycle in concentric than in eccentric type. In the eccentric type, the formation of jet is more prominent for a longer length than in concentric type. Similarly, the flow separation is more intense in downstream side in former case than in later. But high vortex formation is observed to be more in concentric type than in eccentric type, causing severe turbulence. During flow deceleration, the flow gets more complicated due to back flow, causing severe vortex in CCN and complicated flow separation and eddy formation in ECN. Though a simple model is considered for the analysis, the change in flow behavior agrees well with available literature and clinical observations. The results demonstrate that the $3 \mathrm{D}$ stenotic CFD model is capable of predicting the changes in flow behavior for increased severity. Also this study can be further extended to various anatomically realistic models of stenosed carotid artery and predict a range of haemodynamic phenomena that are likely to have clinical significance.

\section{Nomenclature}

$\begin{array}{ll}\text { CCA } & \text { Common Carotid Artery } \\ \text { ECN } & \text { Eccentric type of stenosis } \\ \text { CCN } & \text { Concentric type of stenosis } \\ \text { WSS } & \text { Wall shear stress } \\ \mathrm{V}^{*} & \text { Normalized velocity }=\mathrm{V}_{\mathrm{t}} / \mathrm{V}_{\max }\end{array}$


$\begin{array}{ll}\mathrm{V}_{\mathrm{t}} & \text { Velocity at time, } \mathrm{t} \\ \mathrm{V}_{\max } & \text { Maximum velocity in time period } \\ \mathrm{WSS}^{*} & \text { Normalized WSS }=\mathrm{WSS}_{\mathrm{t}} / \mathrm{WSS}_{\max } \\ \mathrm{WSS}_{\mathrm{t}} & \text { Wall Shear Stress at time, } \mathrm{t} \\ \mathrm{WSS}_{\max } & \text { Maximum Wall Shear Stress in time period }\end{array}$

\section{References}

1. C. A. Taylor, T.J.R Hughes, Christopher K. Zarinsb, Finite element modeling of blood flow in arteries, Comput. Methods Appl. Mech. Engg., 158, 155-196 (1998)

2. Wei Zhang, C. H, Effect of Surrounding Tissue on Vessel Fluid and Solid Mechanics, Transactions of the ASME, 126, 760-769 (2004)

3. G. S. Tavakoli, M.M. Natarajan, Movahedi, Numerical Simulation of pulsatile flow with Newtonian and non-Newtonain behavior in arterial stenosis, Iranian Cardiovascular Research Journal, 1(3), 167-174 (2008)

4. G. Lorenzini and E. Casalena, CFD analysis of pulsatile blood flow in an atherosclerotic human artery with eccentric plaques, J. Biomech., 41, 1862-1870 (2008)

5. Hun Jung, Jong Wook Choi, Chan Guk Park, Asymmetric flows of non-Newtonian fluids in symmetric stenosed artery, Korea-Australia Rheology Journal, 16(2), 101108 (2004)

6. Khalifa A.M.A and D.P Giddens, Characterization and evolution of post-stenotic flow disturbances, J. Bio-mech, 14-5, 279-296 (1981)

7. Mishra J.C and M.K. Patra, A non-Newtonian fluid model for blood flow through arteries under stenotic conditions, J. Bio-mech, 26, 1129-1141 (1993)

8. S. M. Stanley, Transport Phenomena in the Cardiovascular System, WileyInterscience Inc,(1972)

9. W. W Nichols and M.F. O'Rourke, McDonald's Blood Flow in Arteries, LEA \& FEBIGER, Philadelphia, London, UK, 81-85 (1998)

10. E.L. Seung, L. Sang-Wook, F. Paul, S. B. Hisham, L. Francis, "Direct numerical simulation of transitional flow in a stenose carotid bifurcation," J. Biomech., vol.41, 2008, pp 2551-2561.

11. Y.C Fung, Biodynamics- Circulation, Springer Verlag, New York Inc, (1984)

12. J. D. Humphrey and S. L. Delange, An Introduction to Biomechanics, Springer Verlag, New York Inc., (2004)

13. K. Perktold and G. Rappitsch, Computer simulation of local blood flow and vessel mechanics in a compliant carotid artery bifurcation model, J. Biomech., 28, 845-856 (1995)

14. S. Z. Zhao, X. Y. Xu and M. W. Collins, Blood flow and vessel mechanics in physiological realistic model of a human carotid arterial bifurcation, J. Biomech., 32, 975-984 (2000) 
15. ANSYS Inc., ANSYS Release 12.0 Documentation, ANSYS Company, Pittsburgh, PA, (2008). 